\title{
Philosophiques
}

\section{Ockham et la distinction entre les termes abstraits et concrets}

\section{Jennifer Ashworth}

Volume 32, numéro 2, automne 2005

URI : https://id.erudit.org/iderudit/011877ar

DOI : https://doi.org/10.7202/011877ar

Aller au sommaire du numéro

Éditeur(s)

Société de philosophie du Québec

ISSN

0316-2923 (imprimé)

1492-1391 (numérique)

Découvrir la revue

Citer ce document

Ashworth, J. (2005). Ockham et la distinction entre les termes abstraits et concrets. Philosophiques, 32(2), 427-434. https://doi.org/10.7202/011877ar d'utilisation que vous pouvez consulter en ligne.

https://apropos.erudit.org/fr/usagers/politique-dutilisation/ 


\title{
Ockham et la distinction entre les termes abstraits et concrets
}

\author{
JENNIFER ASHWORTH \\ Université de Waterloo \\ ejashwor@watarts.waterloo.ca
}

Quand j'ai lu l'ouvrage magistral de Claude Panaccio, je me suis rendu compte que j'aurais de la difficulté à en discuter, parce que je suis d'accord avec tout ce dit l'auteur, surtout en ce qui concerne les problèmes du langage. Je trouve en particulier décisif les arguments qu'il présente contre les thèses de Paul Spade. Ce dernier a argumenté, en se basant sur trois prémisses, qu'il n'y a pas de terme connotatif simple dans le langage mental. Premièrement, chaque terme connotatif a une définition nominale qui, en principe, ne contient que des termes absolus. Deuxièmement, un terme connotatif est synonyme de sa définition. Troisièmement, il n'y a pas de synonymie dans le langage mental. Il s'ensuit que, dans le langage mental, un terme connotatif sera remplacé par une séquence de termes absolus qui, selon Ockham, réfèrent aux substances et qualités individuelles d'une manière directe. En opposition à Spade, Panaccio a montré qu'il est impossible d'éliminer les concepts connotatifs simples du langage mental et que les termes connotatifs simples ne sont pas synonymes de leurs définitions nominales. Il est vrai que par ses analyses du langage Ockham voulait montrer que l'on pouvait parler du monde sans multiplier les entités, mais on peut atteindre cet objectif tout en admettant une certaine complexité au niveau des concepts simples. En outre, Panaccio a établi deux thèses importantes. D'abord, Ockham ne s'intéresse pas à la construction d'un langage mental idéal mais plutôt au fonctionnement idéal de notre esprit. En deuxième lieu, l'étude de ce fonctionnement idéal ne nous donne pas toutes les solutions aux problèmes de signification parce que, pour comprendre l'acception des termes, il faut connaître les intentions des impositeurs, ceux qui ont donné leur signification primordiale aux termes oraux. Selon Panaccio, Ockham présente une théorie externaliste de la signification du langage.

Il serait inutile de reproduire ici tout ce que Panaccio a déjà expliqué avec une clarté admirable dans son ouvrage, ou d'examiner les mêmes textes que lui. Je me contenterai d'apporter quelques précisions et de signaler dans l'œuvre d'Ockham les passages pertinents qui appuient les conclusions de Panaccio. Afin d'accomplir cette tâche, j'examinerai la distinction entre les termes abstraits et concrets, un thème que Panaccio n'aborde pas dans son ouvrage. Dans la première partie de ma discussion je signalerai un problème lié à la définition d'un terme connotatif; dans la seconde partie, je soulignerai l'importance de l'imposition des termes pour Ockham.

Commençons donc par expliquer ce qui distingue les termes abstraits des termes concrets. Pour un réaliste, la réponse est simple: un terme abstrait 
comme humanitas ( «humanité») nomme une nature commune, et un terme concret, comme homo («homme»), nomme les individus qui participent à cette nature. Pour Ockham, le nominaliste, la différence entre les deux types de termes n'est pas sémantique mais morphologique ${ }^{1}$. Dans le langage oral, un terme concret a une terminaison qui diffère de celle du terme abstrait, et, dans plusieurs cas, il contient moins de syllabes. Il y a aussi une différence grammaticale. Fréquemment, le terme concret est un adjectif et le terme abstrait, un substantif. Cela dit, il convient de constater que le même terme peut faire fonction soit d'abstrait, soit de concret ${ }^{2}$. Par rapport au mot bumanitas, homo est un terme concret, mais par rapport au terme humanum, homo est un terme abstrait.

Dans ses Quodlibeta Ockham demande s'il y a une distinction entre les termes abstraits et concrets dans le langage mental, et, afin de répondre à cette question, il propose une division en quatre groupes ${ }^{3}$. Dans le premier groupe on trouve les termes concrets qui supposent pour et signifient quelque chose que le terme abstrait approprié ne suppose pour ni ne signifie. Ce groupe comporte trois subdivisions. Soit le terme concret suppose pour un sujet et le terme abstrait pour un accident inhérent; soit le terme concret suppose pour un être composite et le terme abstrait pour une partie de cet être; soit les deux termes supposent pour des choses distinctes dont l'une n'est ni sujet ni partie de l'autre. Les trois couples qui illustrent ces subdivisions sont album et albedo («blanc» et «blancheur»), animatum et anima ( «animé» et «âme»), humanum et homo («humain» et «homme»).

Tous les termes concrets qui appartiennent au premier groupe sont dénominatifs. Les termes dénominatifs incluent les paronymes d'Aristote, mais deux constatations s'imposent. Tout d'abord, au sens strict du mot « univoque ", selon lequel les termes univoques sont tous des termes absolus prédiqués in quid, les termes dénominatifs sont opposés aux termes univoques et équivoques, mais au sens large un terme dénominatif est soit univoque, soit équivoque ${ }^{4}$. Deuxièmement, si on précise qu'un terme dénominatif est un terme concret qui signifie un accident intrinsèque et diffère du terme abstrait seulement par sa terminaison, des trois exemples seul album est un terme dénominatif ${ }^{5}$. Ockham préfère varier la première condition (et parfois la seconde), et donc prendre "dénominatif» au sens large, qui inclut animatum et humanum aussi bien que album ${ }^{6}$.

Tous ces termes dénominatifs sont aussi connotatifs. Dans les mots de Panaccio «les connotatifs [...] sont des signes qui présentent une structure

1. SL I, 5, OPh I, p. 16.

2. SL I, 5, OPh I, p. 18.

3. Quodl. V, q. 9, OTh IX, p.513-518; cf. SL I, 5-9, OPh I, p. 16-35.

4. Quodl. IV, q. 12, OTh IX, p.356-357; Exp. in Praedic. 3, OPh II, p. 146.

5. Exp. in Praedic. 3, OPh II, p.144-147; Rep. III, 10, OTh VI, p. 318.

6. Rep. III, 10, OTh VI, p. 318; Ord. I, dist. 2, q. 9, OTh II, p. 330-331. Ockham n'utilise pas l'exemple «humanum» dans ces deux textes. 
sémantique hiérarchisée: outre leur signification première, ils ont une connotation (ou signification seconde ${ }^{7}[. .]$.$» . Chaque connotatif a une définition$ nominale, c'est-à-dire une explication du mot, qui contient, soit un terme in recto et un autre in obliquo, soit un verbe. D'après cette définition, "album » est un terme connotatif avec la définition nominale "aliquid informatum albedine» ou "aliquid habens albedinem ${ }^{8}$. Le signifié premier est la chose qui est blanche, et le signifié second est la qualité individuelle, la blancheur. En principe, ou proprie loquendo, les termes connotatifs n'ont qu'une définition nominale, et en principe les termes absolus comme «bomo» n'ont qu'une définition quid rei, c'est-à-dire, une description des traits essentiels de leurs signifiés ${ }^{9}$.

Jusqu'ici les principes de classification semblent être assez clairs. Or, il est important de noter que le couple standard sanum sanitas ( "sain » "santé ») ne correspond pas à cette classification. On pourrait penser que le couple appartient au premier groupe, et que «sanum» est un nom dénominatif et connotatif qui jouit d'une définition nominale in obliquo, par exemple: "une chose vivante possédant la santé». Cependant, on aurait tort. En ce qui concerne le couple, le texte permet de supposer qu'il appartient au quatrième groupe, plutôt qu'au premier. Les termes concrets de ce groupe, comme "popularis", supposent pour une chose, et les membres abstraits, comme "populus », supposent pour plusieurs choses prises ensemble. Dans son commentaire sur les Catégories, Ockham explique que dans la catégorie de qualité, certains termes signifient des choses simples, sans aucune connotation; certains termes signifient plusieurs choses prises ensemble; et certains termes signifient une chose en connotant une autre ${ }^{10}$. "Sanitas " signifie une relation entre les humeurs, donc elle signifie plusieurs choses prises ensemble, comme le mot "populus»" ${ }^{11}$. Bien que le terme abstrait soit prédiqué de plusieurs choses à la fois, le concret "sanum» est prédiqué d'une seule chose, un animal; par conséquent le terme abstrait n'est pas synonyme du terme concret.

Quant au terme "sanum», Ockham nie qu'il soit connotatif quand il est pris au sens principal. Dans son Ordinatio, comme dans sa Reportatio et ses Quodlibeta, Ockham utilise l'exemple "sanum» dans le contexte de

7. Claude Panaccio, «Guillaume d'Ockham, les connotatifs et le langage mental», Documenti e Studi Sulla Tradizione Filosofica Medievale 11 (2000), p. 298.

8. SL I, 10, OPh I, p. 36. "Nomen autem connotativum est illud quod significat aliquid primario et aliquid secundario. Et tale nomen proprie habet definitionem exprimentem quid nominis, et frequenter oportet ponere unum illius definitionis in recto et aliud in obliquo. [...] Unde si quaeras, quid significet hoc nomen 'album', dices quod illud idem quod ista oratio tota 'aliquid informatum albedine' vel 'aliquid habens albedinem'."

9. $S L$ I, 10, OPh I, p. 35-36. Voir $S L$ III-2, 28, OPh I, p. 555-556 et $S L$ III-2, 33, OPh I, p. 568-569 pour les usages impropres des définitions quid rei et nominales.

10. Exp. in Praedic., 14, OPh II, p. 270.

11. Exp. in Praedic., 14, OPh II, p. 287-288. 
l'analogie, qui pour Ockham est un type d'équivocité à dessein. L'exemple est important pour lui parce que, selon Aristote, au moins dans les traductions latines, le mot «ens» est dit medio modo, c'est-à-dire analogice, comme le mot «sanum $»^{12}$, tandis que pour Ockham le mot «ens» est univoque ${ }^{13}$. Il suggère que pour les Grecs le mot "ens» était équivoque et que la comparaison entre «ens» et «sanum» était correcte pour eux, bien qu'elle ne le soit pas pour nous ${ }^{14}$. Dans la Reportatio, il prétend que l'on prédique le mot "sanum» univoquement et in quid d'un animal mais dénominativement, parce que connotativement, d'une diète et de l'urine ${ }^{15}$. Dans l'Ordinatio, Ockham prétend que «sanum», prédiqué d'un animal, signifie la qualité de santé absolute, ne connotant rien d'autre sauf peut être le sujet (nibil aliud nisi forte subiectum connotando), et il ajoute que prédiqué d'un animal le mot n'est pas un nom connotatif, bien qu'il le soit quand il est prédiqué de la diète ou de l'urine ${ }^{16}$. Dans ces derniers cas, le terme jouit d'une définition nominale in obliquo. Une diète saine est la cause de la santé d'un animal, et l'urine saine est un signe de la santé d'un animal. Cette discussion soulève la question: comment le terme "sanum » suppose-t-il quand il est prédiqué d'un animal ou quand il est le sujet d'une proposition, étant donné qu'il n'est pas un terme connotatif, et que la vérité des propositions "animal est sanum» et «sanum currit» requiert que le sujet et le prédicat supposent pour le même individu? Ce que Guillaume dit à propos de «sanum» ne correspond pas à la définition de sa signification au sens strict, selon lequel un terme pris en supposition personnelle signifie et suppose pour le même individu ${ }^{17}$, ni à sa discussion des termes connotatifs et dénominatifs. Il semble que la distinction entre termes absolus et connotatifs ne soit pas aussi claire que Panaccio le pense.

12. Quodl. IV, q. 12, OTh IX, p. 356; Rep. III, 10, OTh VI, p. 326-327. Cf. Ord. I, dist. 2, q. 9, OTh II, p. 325.

13. Quodl. IV, q. 12, OTh IX, p. 358-359; Ord. I, dist. 2, q. 9, OTh II, p. 334-336; Rep. III, 10, OTh VI, p. 340.

14. Quodl. IV, q. 12, OTh IX, p. 357-358; Ord. I, dist. 2, q. 9, OTh II, p. 334-335. Cf. Rep. III, 10, OTh VI, p. 340.

15. Rep. III, 10, OTh VI, p. 339-340.

16. Ord. I, dist. 2, q. 9, OTh II, p.327-328. «Primum membrum potest subdividi multipliciter: uno modo dicitur tale aequivocum quando idem sub eisdem modis grammaticalibus significat ista I diversa, sicut 'homo' est aequivocum ad hominem vivum et ad hominem pictum; alio modo quando significat diversa diversis modis, quia respectu unius significati est nomen connotativum et respectu alterius non est connotativum. Exemplum de sano, quia sanitas [om. dans un manuscrit] quando significat qualitatem formaliter exsistentem in animali non est nomen connotativum; quando autem significat diaetam vel urinam tunc est nomen connotativum, quia quando significat aliquid illorum, significat unum illorum in recto et primum significatum suum in obliquo, quia si deberet exprimi quid nominis, aliquod illorum deberet poni in recto et sanitas in obliquo, quia sanum quod dicitur de diaeta habet illud quid nominis 'sanum est diaeta effectiva sanitatis'; secundum autem quod dicitur de urina habet istud quid nominis 'sanum est urina significans sanitatem'. »

17. SL I, 33, OPh I, p. 95; SL I, 64, OPh I, p. 195. 
Passons donc à la deuxième partie de ma discussion, qui à trait aux problèmes de synonymie et d'imposition dans le contexte des termes abstraits et concrets. Le deuxième groupe de ces termes et le seul qui ne se retrouve pas dans le langage mental est celui dont les membres abstraits sont synonymes des membres concrets, et le troisième groupe est celui dont les membres abstraits ne sont pas synonymes des concrets à cause des syncatégorèmes ou des déterminations adverbiales que les termes abstraits renferment. Comme nous allons le voir, les mêmes couples sont en jeu dans les deux cas.

Tout d'abord, il convient de regarder la synonymie de plus près. Pour les grammairiens et les logiciens du moyen âge, la synonymie est un rapport entre deux mots oraux ou écrits. L'exemple standard, trouvé chez Boèce et Priscien, est celui de l'épée qu'on peut nommer "gladius», "mucro», ou «ensis ${ }^{18}$. Le choix entre ces mots ne peut être motivé que par le désir de produire un discours élégant. Quand Ockham nie qu'il y ait de la synonymie dans le langage mental, il est souvent évident qu'il ne pense qu'aux exemples standards ${ }^{19}$. Cependant, il y a trois textes importants qui donnent une définition de synonymie en fonction des modes grammaticaux et logiques ${ }^{20}$, et en fonction des intentions des impositeurs et des locuteurs de la langue. Dans son Ordinatio, dans un passage ajouté, Ockham donne deux définitions: soit les noms synonymes signifient les mêmes individus sous les mêmes modes, soit les impositeurs et les locuteurs ont l'intention que les mots signifient les mêmes individus sous les mêmes modes ${ }^{21}$. Dans la Summa Logicae et les Quodlibeta, il nuance la première définition: les noms synonymes signifient les mêmes individus sous les mêmes modes, même s'il y a des locuteurs qui pensent à tort que les noms ne sont pas synonymes ${ }^{22}$.

Commençons par examiner le couple «deus» et «deitas». Si ces noms sont synonymes, comment le locuteur peut-il se tromper? David Chalmers a expliqué l'erreur du locuteur en proposant qu'il possède deux concepts

18. Boèce, In Categorias Aristotelis libri quatuor, PL 64 (Paris, 1891), col. 164A; Priscien, Institutiones grammaticae, éd. M. Hertz (Leipzig, 1855; reprint Hildesheim, New York, 1981) t. 1, II. 26, p. 59: 17-19. I, p. 20.

19. Quodl. V, q. 8, OTh IX, p.510-511; Quodl. V, q. 9, OTh IX, p. 513; SL I, 6, OPh

20. Il est à noter que les modi significandi, surtout les modes grammaticaux, peuvent varier sans détruire la synonymie. C'est surtout les deux modes logiques, «signifier directement » et «signifier indirectement" ou "connoter", qui comptent: SL I, 72, OPh I, p. 223-224. Cf. Quodl. V, q. 9, OTh IX, p. 518; et SL I, 33, OPh I, p. 96, sur "significare communissime».

21. Ord. I, dist. 2, q. 3, OTh II, p. 88.

22. SL I, 6, OPh I, p. 19;Quodl. V, q. 10, OTh IX, p. 519: "Large dicuntur illa nomina synonyma quae significant simpliciter idem omnibus modis et eodem modo significandi, ita quod nibil aliquo modo significatur per unum quin eodem modo significandi significetur per reliquum, quamvis non omnes utentes credant ipsa idem significare, sed decepti aestimant aliquid significari per unum quod non significatur per reliquum; sicut si aliquis crederet quod hoc nomen 'Deus' importaret unum totum et 'Deitas' partem eius. » 
distincts mais synonymes ${ }^{23}$. Cependant, dans les Quodlibeta et la Summa Logicae, Ockham propose que le locuteur pense que «deus» nomme un tout, et que "deitas» nomme une partie du tout ${ }^{24}$. D'après moi, cela veut dire que le locuteur, à cause d'une opinion erronée quant à l'imposition originale des mots, a construit deux concepts non synonymes. On pourrait aussi avancer l'hypothèse que le locuteur possède un seul concept, sans le reconnaître. Dans son Ordinatio, Ockham avait déjà discuté de la difficulté de savoir si on possède un concept univoque, c'est-à-dire, un concept un ${ }^{25}$. On peut faire appel à l'expérience, en essayant de formuler une proposition dans l'esprit avec un sujet ou un prédicat qui suppose en même temps pour tous les individus que le terme signifie, et cela sine omni lingua et idiomate. On peut aussi faire appel à l'argumentation, en essayant de réfuter les arguments contre l'existence du concept univoque. Cependant, Ockham concède que ces deux méthodes sont difficiles à mettre en application, ce qui donne à penser qu'un locuteur ne serait pas toujours en mesure de décider s'il possède un concept ou deux.

Dans son Ordinatio, Ockham nous offre une discussion plus soutenue du problème de la synonymie du couple «deus» "deitas», et il nous offre deux hypothèses à propos de l'imposition primordiale des deux termes ${ }^{26}$. Premièrement, l'impositeur aurait pu instituer les termes à signifier par le biais de deux concepts composés, tels qu'une partie d'un concept convient à quelque chose auquel une partie de l'autre concept ne convient pas. Par exemple, il aurait pu définir "deus» comme "aliquod ens intellectuale» et "deitas" comme "ens quod est sapientia». Ou bien l'impositeur aurait pu instituer les mots à signifier d'après le modèle des termes concrets qui signifient les choses composites, et leurs abstraits. Dans ce cas, et la formation des concepts de Dieu et l'imposition des noms dépendent de la conviction que les termes abstraits et concrets ont des signifiés distincts, et sans cette conviction les noms auraient été synonymes ${ }^{27}$. En conséquence, on peut argumenter que les noms «deus» et «deitas» ne sont pas synonymes; et, qui plus est, on peut argumenter qu'on n'est pas en mesure de savoir si les noms «bomo» et «humanitas» sont synonymes, car on peut douter de l'imposition primordiale.

23. David Chalmers, «Is There Synonymy in Ockham's Mental Language?», in The Cambridge Companion to Ockham, éd. Paul Vincent Spade (Cambridge, 1999), p. 88.

24. Quodl. V, q. 10, OTh IX, p. 519; SL I, 6, OPh I, p. 19.

25. Ord. I, dist. 29, q. un, OTh IV, p. 277-278.

26. Ord. I, dist. 2, q. 3, OTh II, p. 85-89.

27. Ord. I, dist. 2, q. 3, OTh II, p. 87: «[...] intellectus potest formare duos conceptus in quibus quasi intelligitur Deus, quia aliter a nobis non potest intelligi, et hoc secundum similitudinem ad duos conceptus, quorum unus est concretus et alius abstractus, formatos de re composita, ita quod nisi inveniret in rebus compositis concretum et abstractum distingui quoad signatum, non formaret tales conceptus de Deo nec imponeret talia nomina nisi sicut nomina synonyma."; ibid., p.88-89: "et isto modo homo et humanitas, Deus et deitas non sunt synonyma, et hoc quia relinquunt in dubio quantum est ex impositione istorum nominum an haec sit vera 'homo est humanitas' vel falsa, et ita de aliis. " 
Considérons maintenant ce couple «homo» «humanitas» de plus près. Ockham est convaincu que pour Aristote ces termes sont synonymes ${ }^{28}$, mais il reconnait que cette synonymie pose des problèmes pour la théologie et pour le langage quotidien. On ne dit pas «bumanitas est homo» ni «humanitas est $a l b a »$. En théologie, le problème concerne le Christ, la deuxième personne de la Trinité, qui est une personne (suppositum) avec deux natures, humaine et divine. Dans le cas des hommes créés, on ne peut pas faire une distinction entre la nature humaine et un individu humain, mais dans le cas du Christ, on ne peut pas identifier la personne divine à la nature humaine. Donc, dans les Quodlibeta et la Summa Logicae, Ockham fait appel à deux définitions nominales ${ }^{29}$. «Humanitas» signifie une nature faite d'un corps et d'une âme intellective, sans connoter que cette nature peut (ou ne peut pas) être assumée par une personne divine. "Homo» signifie, soit une nature composée d'un corps et d'une âme intellective qui n'est pas soutenue par un suppositum distinct, soit un suppositum distinct qui soutient une telle nature. La première partie de la disjonction suppose pour Socrate, la seconde pour le Christ. On est maintenant en mesure de résoudre les problèmes de langage en substituant leurs définitions nominales aux termes abstraits ou concrets. Cette procédure nous permet de statuer sur la vérité des propositions en question, et de nier «Omnis homo est humanitas» tout en concédant les deux propositions «Homo est humanitas» et «Homo non est humanitas». Ockham conclut que le cas théologique montre qu'il n'y a pas de synonymie, même si on nie la présence de syncatégorèmes ${ }^{30}$. On peut remarquer aussi que le terme absolu «homo » est maintenant considéré comme dénominatif, car il connote le suppositum divinum ${ }^{31}$.

Dans le cas du langage quotidien, Ockham fait appel aux syncatégorèmes que les termes abstraits renferment ${ }^{32}$. Là où Claude Panaccio parle des abréviations, je préfère suivre le texte des Quodlibeta et parler des définitions contextuelles. Évidemment, Ockham n'utilise pas cette phrase, mais il stipule que la substitution d'une abréviation à un terme abstrait doit se produire dans une proposition. Il explique qu'en vertu de l'imposition ou de l'emploi du terme "humanité» par les locuteurs, la proposition «L'humanité est blanche" (Humanitas est alba) veut dire "L'homme en tant qu'homme est blanc» ou «L'homme est nécessairement [per se] blanc » ${ }^{33}$. Dans la Summa

28. Quodl. V, q. 11, OTh IX, p. 525; SL I, 6, OPh I, p. 20.

29. Quodl. V, q. 10, OTh IX, p. 520; SL I, 7, OPh I, p. 25.

30. SL I, 7, OPh I, p. 24-25.

31. Rep. III, 10, OTh VI, p. 321-322.

32. Quodl. V, q. 9, OTh IX, p. 516; SL I, 8, OPh I, p. 29-34.

33. Quodl. V, q. 9, OTh IX, p. 516: "Tertius modus est quando abstractum et concretum sic se habent quod abstractum ad placitum instituentium vel ex usu loquentium includit aliqua syncategoremata vel determinationes adverbiales, ita quod illud abstractum in significando aequivaleat unum complexum; puta 'humanitas' tantum valet sicut 'homo in quantum' vel 'necessario' vel 'contingenter' vel 'per se', quando ponitur in propositionibus; concretum autem nullum tale syncategorema includit. Et secundum istam viam tantum valet ista 'humanitas est alba' sicut 'homo in quantum homo est albus' vel 'homo per se est albus'.» 
Logicae, il ajoute que de tels termes abstraits sont rarement utilisés dans le langage vulgaire, mais qu'ils sont fréquents chez les philosophes et les Sancti ${ }^{34}$.

Pour terminer, je ferai deux remarques. Premièrement, sans tomber dans l'erreur ontologique, deux personnes peuvent avoir des concepts différents, tout en utilisant les mêmes mots oraux. Rappelons le cas du couple «homo» "humanitas": on peut penser soit comme les théologiens, soit comme Aristote (pourvu qu'on ne parle pas de théologie), soit comme ceux qui préfèrent une définition contextuelle du terme abstrait. Deuxièmement, on peut se trouver dans une situation où on ignore la signification d'un terme, parce qu'on ne sait pas quelle définition l'impositeur original a utilisé. Panaccio a raison quand il propose qu'Ockham emploie une théorie externaliste de la signification. Quand on veut établir l'acception d'un terme, ce n'est pas ses propres concepts qu'il faut consulter, mais les concepts de l'impositeur. En ce qui concerne le langage de tous les jours, on peut sans problème suivre sa communauté linguistique, et ses propres expériences, mais en ce qui concerne le langage théologique ou philosophique, même une communauté linguistique sophistiquée peut tomber dans l'erreur.

\section{Abréviations}

Exp. in Praedic. Expositio in Librum Praedicamentorum Aristotelis, G. Gál (dir.), in Opera Philosophica II. St. Bonaventure, N.Y., St. Bonaventure University, 1978.

Ord., OTh II Scriptum in Librum Primum Sententiarum Ordinatio. Distinctiones II-III, S. Brown et G. Gál (dir.). Opera Theologica II. St. Bonaventure, N.Y., St. Bonaventure University, 1970.

Ord., OTh IV Scriptum in Librum Primum Sententiarum Ordinatio. Distinctiones XIX-XLVIII, G. Etzkorn et F. E. Kelley (dir.). Opera Theologica IV. St. Bonaventure, N.Y., St. Bonaventure University, 1979.

Quodl. Quodlibeta Septem, J. C. Wey (dir.). Opera Theologica IX. St. Bonaventure, N.Y., St. Bonaventure University, 1980.

Rep. Quaestiones in Librum Tertium Sententiarum (Reportatio), F. E. Kelley et G. I. Etzkorn (dir.). Opera Theologica VI. St. Bonaventure, N.Y., St. Bonaventure University, 1982.

$S L$ Summa Logicae, P. Boehner, G. Gál et S. Brown (dir.). Opera Philosophica I. St. Bonaventure, N.Y., St. Bonaventure University, 1974. 